\title{
POSTNIKOV SECTIONS OF FORMAL AND HYPERFORMAL SPACES
}

\author{
SAMUEL B. SMITH
}

(Communicated by Thomas Goodwillie)

\begin{abstract}
Let $X$ be a simply connected CW complex and $X_{n}$ its $n$th Postnikov section. We prove that $X$ is formal provided $H^{q}\left(X_{n}, \mathbb{Q}\right)$ is additively generated by decomposables for all $q$ and $n$ with $q>n$. Recall from [4] that a space $X$ is said to be hyperformal if its rational cohomology algebra is the quotient of a free graded algebra by an ideal generated by a regular sequence. Using the main result of Felix and Halperin's paper (Trans. Amer. Math. Soc. 270 (1982), 575-588) we show our sufficient condition for formality is actually equivalent to hyperformality.
\end{abstract}

\section{INTRODUCTION}

A formal space, imprecisely stated, is one whose rational homotopy type is a formal consequence of its rational cohomology algebra. Since their emergence from Sullivan's theory of minimal models, formal spaces have been shown to enjoy many desirable properties (see, e.g., [1]) and much effort has been made to characterize these spaces (see, e.g., $[5,7,8]$ ). The first and most basic characterization of formal spaces was given by Deligne et al. [3] and states that a space is formal if and only if all (rational) Massey and higher-order products vanish uniformly. In [4], Felix and Halperin prove that a simply connected formal space with finite-dimensional rational homotopy actually satisfies the stronger condition of hyperformality; that is, its rational cohomology algebra is the quotient of a free graded algebra by an ideal generated by a regular sequence. The purpose of this paper is to provide a characterization of hyperformal spaces in the spirit of Deligne et al.

In this paper we take the view that nontrivial Massey and higher-order products of $X$ correspond to unexpected indecomposable elements in the rational cohomology of Postnikov sections of $X$; indecomposables which occur in a degree greater than the dimension of the highest nontrivial rational homotopy group of a Postnikov section. With this perspective and the characterization of formality given by Deligne et al., it seems plausible that a space is formal provided $H^{q}\left(X_{n}, \mathbb{Q}\right)$ is additively generated by decomposable elements for all

Received by the editors November 10, 1992 and, in revised form, February 8, 1993.

1991 Mathematics Subject Classification. Primary 55P62.

Key words and phrases. Rational homotopy theory, formality, hyperformality, minimal model. 
$q>n$ where $X_{n}$ is the $n$th Postnikov section of $X$. This assertion is the content of our first main result (Theorem 4.1). On the other hand, it is easy to check (see Example 4.2) that if $X_{3}$ is the third Postnikov section of the formal space $S^{2} \vee \mathbb{C} P^{\infty}$ then $H^{5}\left(X_{3}, \mathbb{Q}\right)$ contains an indecomposable element. In some sense, the Massey and higher-order products which appear as unexpected indecomposables in the cohomology of Postnikov sections of $S^{2} \vee \mathbb{C P}$ are killed later by $k$-invariants of $S^{2} \vee \mathbb{C} P^{\infty}$. Implicit in the Felix and Halperin result is the fact that this phenomenon-all unexpected indecomposables are killed by later $k$-invariants-requires the space to have infinitely many rational homotopy groups. In fact, using their result we prove in $\S 4$ that for simply connected spaces with finite-dimensional rational homotopy our condition is equivalent to formality. More generally, we prove that for all spaces of finite rational type our condition is equivalent to hyperformality.

The paper is organized as follows. Section 2 is preliminary; we review Sullivan's theory of minimal models, give precise definitions of formal and hyperformal spaces, and then recall the characterization given the former by Deligne et al. In $\S 3$, we consider two-stage minimal models and prove that our condition is sufficient for formality for spaces whose minimal models are of this form. Section 4 contains the statements and proofs of the main results. Throughout this paper all spaces will be taken to be simply connected CW complexes with finite-dimensional rational homotopy groups in each degree. Similarly, all graded algebras will be simply connected and of finite type.

I would like to thank my thesis advisor, Professor Donald Kahn, for his assistance in connection with this work.

\section{Preliminaries}

In this section we recall results and establish notation for working with differential graded algebras (DGAs) and Sullivan's theory of minimal models. We then define formal and hyperformal spaces and give the characterization of the former in terms of Massey and higher-order products. Our references for this material are $[2,3,9]$.

By a DGA $(\mathscr{A}, d)$ we will mean a simply connected commutative graded algebra $\mathscr{A}$ over $\mathbb{Q}$ together with a degree one derivation $d$ whose square is zero. Given a rational vector space $V_{i}$ we denote by $\Lambda_{i}\left(V_{i}\right)$ the free graded algebra generated in degree $i>1$ by the elements of $V_{i}$. A DGA $\mathscr{A}$ is free if $\mathscr{A}$ may be written in the form $\mathscr{A}=\bigotimes_{i=2}^{\infty} \Lambda_{i}\left(V_{i}\right)$. In this case, we will write $\mathscr{A}=\Lambda(Z)$ where $Z$ is the graded vector space $Z=\bigoplus_{i=2}^{\infty} V_{i}$.

Suppose $(\mathscr{A}, d)$ is a DGA and $Z$ is a graded vector space. Then we may form a new DGA $\left(\mathscr{A}^{\prime}, d^{\prime}\right)$ which we denote by $\mathscr{A}^{\prime}=\mathscr{A} \otimes_{d^{\prime}} \Lambda(Z)$, where, as a graded algebra, $\mathscr{A}^{\prime}=\mathscr{A} \otimes \Lambda(Z)$, and where the differential $d^{\prime}$ is defined by $d^{\prime} \mid \mathscr{A}=d$, while $d^{\prime}$ on $\Lambda(Z)$ is some degree one derivation from $\Lambda(Z)$ to the closed elements of $(\mathscr{A}, d)$. Note that $d^{\prime}$ determines a degree one linear map $d^{\prime} \mid Z: Z \rightarrow H^{*}(\mathscr{A}, d)$.

A (simply connected) DGA $(\mathscr{K}, d)$ is minimal if $\mathscr{M}$ is free as a graded algebra and if the differential $d$ takes values in the decomposable elements of $\mathscr{M}$. In Sullivan's theory of minimal models, to each simply connected complex $X$ there is assigned a minimal DGA $\left(\mathscr{M}(X), d_{X}\right)$ such that $H^{*}\left(\mathscr{M}(X), d_{X}\right) \cong$ 
$H^{*}(X, \mathbb{Q})$ as algebras. The minimal DGA $\left(\mathscr{M}(X), d_{X}\right)$ is unique up to DGA isomorphism. Moreover, $\left(\mathscr{M}(X), d_{X}\right)$ is a unique invariant of the rational homotopy type of $X$. The minimal DGA $\left(\mathscr{M}(X), d_{X}\right)$ is called the minimal model of $X$.

Given a map $f: X \rightarrow Y$ between simply connected complexes, there is a corresponding DGA map $\mathscr{M}(f): \mathscr{M}(Y) \rightarrow \mathscr{M}(X)$ such that the map induced on cohomology by $\mathscr{M}(f)$ is just $f^{*}: H^{*}(Y, \mathbb{Q}) \rightarrow H^{*}(X, \mathbb{Q})$. The assignment $f \mapsto \mathscr{M}(f)$ sets up $\mathscr{M}$ as a contravariant functor from the rational homotopy category of simply connected complexes and maps to the category of minimal DGAs and homotopy classes of DGA maps.

There is an important relationship between minimal DGAs and rational Postnikov towers which is contained in [3, Lemma 3.2]. Let $(\mathscr{M}, d)$ be a minimal DGA and denote by $\mathscr{M}^{(n)}$ the subalgebra of $\mathscr{M}$ generated by elements of degree $\leq n$. Since $d$ is decomposable, it is easy to see that if $d_{n}=d \mid \mathscr{M}^{(n)}$ then $\left(\mathscr{M}^{(n)}, d_{n}\right)$ is a sub-DGA of $(\mathscr{K}, d)$. Observe that $\left(\mathscr{M}^{(n+1)}, d_{n+1}\right)$ is of the form $\mathscr{M}^{(n+1)}=\mathscr{M}^{(n)} \otimes_{d_{n+1}} \Lambda_{n+1}\left(V_{n+1}\right)$. The differential $d_{n+1}$ determines a linear map $d_{n+1} \mid V_{n+1}: V_{n+1} \rightarrow H^{n+2}\left(\mathscr{M}^{(n)}, d_{n}\right)$. Corresponding to the inclusion $\mathscr{M}^{(n)} \hookrightarrow \mathscr{M}^{(n+1)}$ there is a principal fibration $p_{n+1}: X_{n+1} \rightarrow X_{n}$ with fibre $K\left(V_{n+1}^{*}, n+1\right)$ (where $\left.V_{n+1}^{*}=\operatorname{Hom}\left(V_{n+1}, \mathbb{Q}\right)\right)$ such that the minimal models of $X_{n+1}$ and $X_{n}$ are $\left(\mathscr{K}^{(n+1)}, d_{n+1}\right)$ and $\left(\mathscr{K}^{(n)}, d_{n}\right)$, respectively. The $k$-invariant of this fibration is an element

$$
k_{n+2} \in H^{n+2}\left(X_{n}, V_{n+1}^{*}\right) \cong \operatorname{Hom}\left(V_{n+1}, H^{n+2}\left(\mathscr{M}^{(n)}, d_{n}\right)\right) \text {. }
$$

Viewed in the latter set, the $k$-invariant coincides with $d_{n+1} \mid V_{n+1}$. In particular, we have that if $(\mathscr{M}, d)$ is the minimal model of a simply connected complex $X$, then the minimal model of the $n$th Postnikov section $X_{n}$ of $X$ is just $\left(\mathscr{M}^{(n)}, d_{n}\right)$.

We next give the definition of a formal DGA and a formal space.

Definition 2.1 [3, p. 260]. A DGA $(\mathscr{A}, d)$ is formal if there is a DGA map $\lambda:(\mathscr{A}, d) \rightarrow\left(H^{*}(\mathscr{A}, d), 0\right)$ from $(\mathscr{A}, d)$ to its cohomology viewed as a DGA with trivial differential, such that $\lambda$ induces an isomorphism on cohomology. A space $X$ is formal if its minimal model is formal.

To define hyperformality we must first recall the definition of a regular sequence (see [6, p. 84]). Let $H$ be a graded algebra. A sequence of elements $\alpha_{1}, \alpha_{2}, \ldots$ of $H$ is said to be a regular sequence of $H$ if, for each $i=1,2 \ldots$, the image of $\alpha_{i+1}$ in the factor algebra $H / I\left(\alpha_{1}, \ldots, \alpha_{i}\right)$ is not a zero divisor, where $I\left(\alpha_{1}, \ldots, \alpha_{i}\right)$ denotes the ideal of $H$ generated by $\alpha_{1}, \ldots, \alpha_{i}$. We will assume, moreover, that the elements $\alpha_{i}$ are homogeneous of positive degree. Notice that, in particular, the $\alpha_{i}$ must all be of even degree.

Definition 2.2 [4, p 576]. A graded algebra $H$ is said to be hyperformal if $H$ is of the form $\Lambda(Z) / I\left(\alpha_{1}, \alpha_{2}, \ldots\right)$, where $Z$ is some graded vector space over $\mathbb{Q}$ and $\alpha_{1}, \alpha_{2}, \ldots$ is a regular sequence in $\Lambda(Z)$. A space $X$ is hyperformal if its rational cohomology algebra $H^{*}(X, \mathbb{Q})$ is hyperformal.

We conclude this section by recalling the characterization of formality in terms of Massey and higher-order products. Suppose $(\mathscr{M}, d)$ is a minimal 
DGA. Since $\mathscr{M}$ is a free graded algebra, we may write $\mathscr{M}=\bigotimes_{i=2}^{\infty} \Lambda_{i}\left(V_{i}\right)$. Let $C_{i}$ be the subspace of closed elements in $V_{i}$. Then we have

Lemma 2.3 [3, Theorem 4.1]. $(\mathscr{M}, d)$ is formal if and only if there is in each $V_{i}$ a complement $N_{i}$ to $C_{i}, V_{i}=C_{i} \oplus N_{i}$, such that any closed element in the ideal $I\left(\bigoplus_{i=2}^{\infty} N_{i}\right)$ is exact.

Lemma 2.3 admits the following interpretation. The closed elements in the ideal $I\left(\bigoplus_{i=2}^{\infty} N_{i}\right)$ correspond to Massey or higher-order products in the cohomology of $(\mathscr{M}, d)$. The lemma asserts that $(\mathscr{M}, d)$ is formal if and only if these products vanish uniformly [3, p. 262].

\section{TWO-STAGE MiNimal MODEls}

Let $(\mathscr{M}, d)$ be a minimal DGA of the form $\mathscr{M}=\Lambda\left(Z_{0}\right) \otimes_{d} \Lambda\left(Z_{1}\right)$ (see $\S 2$ ), where $Z_{0}$ and $Z_{1}$ are graded vector spaces and $\Lambda\left(Z_{0}\right)$ is viewed as a DGA with trivial differential. Observe that, since $\mathscr{M}$ is minimal, the differential $d$ is determined by some degree one linear map from $Z_{1}$ into the decomposable elements of $\Lambda\left(Z_{0}\right)$. We refer to DGAs $(\mathscr{M}, d)$ in this form as two-stage minimal DGAs.

Our purpose in this section is to prove that our condition involving decomposables in the rational cohomology of Postnikov sections of a space $X$ is a sufficient condition for $X$ to be formal; provided the minimal model of $X$ is a two-stage minimal DGA. This is the content of Lemma 3.4. We begin with two lemmas regarding the differential $d$ in a two-stage minimal DGA $(\mathscr{M}, d)$.

Observe that we may decompose $\mathscr{M}$ as graded vector space in the form

$$
\mathscr{M}=\Lambda\left(Z_{0}\right) \oplus I\left(Z_{1}\right),
$$

where $I\left(Z_{1}\right)$ denotes the ideal of $\mathscr{M}$ generated by $Z_{1}$. Our first lemma describes the differential $d$ with respect to this decomposition. We prove

Lemma 3.2. Let $\beta \in \mathscr{M}$. Then $\beta=\beta_{0}+\beta_{1}$ where $d\left(\beta_{0}\right) \in \Lambda\left(Z_{0}\right)$ and $d\left(\beta_{1}\right) \in$ $I\left(Z_{1}\right)$.

Proof. Let $c \in \Lambda\left(Z_{0}\right)$ be a homogeneous element, and let $b \in \Lambda\left(Z_{1}\right), \quad b \neq 1$, be homogeneous of the form $b=\prod_{i} z_{i}$, for some $z_{i} \in Z_{1}$. Then $c \otimes b \in I\left(Z_{1}\right)$ and, in fact, the generic element of $I\left(Z_{1}\right)$ is a sum of elements of this form. Since $d \mid \Lambda\left(Z_{0}\right)=0$, it suffices to show that $d(c \otimes b) \in \Lambda\left(Z_{0}\right) \cup I\left(Z_{1}\right)$.

Since $c \in \Lambda\left(Z_{0}\right), \quad d(c)=0$. Thus $d(c \otimes b)= \pm c \otimes d(b)$. If $b \in Z_{1}$ (i.e., $b$ is indecomposable) then $d(b) \in \Lambda\left(Z_{0}\right)$ so, in this case, $d(c \otimes b) \in \Lambda\left(Z_{0}\right)$. Otherwise, $b$ is of the form $b=\prod_{i=1}^{k} z_{i}$, with $k \geq 2$, and so

$$
d(c \otimes b)= \pm c \otimes d(b)= \pm c \otimes\left(d\left(z_{1}\right) \cdot z_{2} \cdots z_{k} \pm \cdots \pm z_{1} \cdots z_{k-1} \cdot d\left(z_{k}\right)\right) .
$$

Since each $d\left(z_{i}\right) \in \Lambda\left(Z_{0}\right)$, we see that, in this case, $d(c \otimes b) \in I\left(Z_{1}\right)$.

Now given the form of $(\mathscr{M}, d)$, we may suppose that $d \mid Z_{1}$ is injective; otherwise we augment $Z_{0}$ by the kernel of $d \mid Z_{1}$. However, given a homogeneous element $z \in Z_{1}$, it is conceivable that $d(z)=d(\beta)$ for some decomposable element $\beta \in \mathscr{M}$. Lemma 3.3 implies that we may replace $(\mathscr{M}, d)$ by an isomorphic two-stage minimal DGA such that this situation does not occur. As the proof shows, this replacement is algebraically analogous to requiring all 
inessential $k$-invariants of a rational space $X_{0}$ (viewed as maps) to be actually constant. Since it is no more difficult, we prove Lemma 3.3 for general minimal DGAs.

Lemma 3.3. Let $(\mathscr{M}, d)$ be any minimal DGA. Then there is an isomorphic minimal $D G A(\mathscr{N}, \delta)$ such that as a free graded algebra $\mathscr{N}=\Lambda(W)$ where if $w \in W$ with $\delta(w) \neq 0$ then $\delta(w) \neq \delta(\beta)$ for any decomposable element $\beta \in \mathscr{N}$. If $(\mathscr{M}, d)$ is a two-stage minimal $D G A$ then $(\mathscr{N}, \delta)$ may be taken to be one also.

Proof. We construct $(\mathcal{N}, \delta)$ and the isomorphism $\varphi: \mathscr{M} \rightarrow \mathcal{N}$ inductively. Define $\mathscr{N}^{(2)}=\mathscr{M}^{(2)}$, and let $\varphi_{2}: \mathscr{M}^{(2)} \rightarrow \mathscr{N}^{(2)}$ be the identity. Suppose we have constructed $\left(\mathscr{N}^{(n)}, \delta_{n}\right)$, a minimal DGA generated by elements of degree $\leq n$, with the required property and a DGA isomorphism $\varphi_{n}: \mathscr{M}^{(n)} \rightarrow \mathscr{N}^{(n)}$. We show how to construct $\left(\mathscr{N}^{(n+1)}, \delta_{n+1}\right)$ and a DGA isomorphism $\varphi_{n+1}$ : $\mathscr{M}^{(n+1)} \rightarrow \mathscr{N}^{(n+1)}$ such that $\left(\mathscr{N}^{(n)}, \delta_{n}\right)$ is the sub-DGA of $\left(\mathscr{N}^{(n+1)}, \delta_{n+1}\right)$ generated by elements of degree $\leq n$ and $\varphi_{n+1} \mid \mathscr{M}^{(n)}=\varphi_{n}$.

As algebras, $\mathscr{M}$ and $\mathscr{N}^{(n)}$ are of the form $\mathscr{M}=\Lambda(Z), \mathscr{N}=\Lambda(W)$ where $Z$ and $W$ are graded vector spaces with $W$ having no elements above degree $n$. Let $Z^{n+1}$ denote the elements of degree $n+1$ in $Z$. We construct an intermediary DGA $\left(\mathscr{A}^{(n+1)}, d_{n+1}^{\prime}\right)$ as follows. Put

$$
\mathscr{A}^{(n+1)}=\mathscr{N}^{(n)} \otimes_{d_{n+1}^{\prime}} \Lambda_{n+1}\left(Z^{n+1}\right),
$$

where $d_{n+1}^{\prime}$ is defined by $d_{n+1}^{\prime}(w)=\varphi_{n}(d(w))$ for $w \in Z^{n+1}$. Notice that if we define $\varphi_{n+1}^{\prime}: \mathscr{M}^{(n+1)} \rightarrow \mathscr{A}^{(n+1)}$ by letting $\varphi_{n+1}^{\prime}$ equal $\varphi_{n}$ on $\mathscr{M}^{(n)}$ and the identity on $Z^{n+1}$, then $\varphi_{n+1}^{\prime}$ is a DGA isomorphism between $\left(\mathscr{M}^{(n+1)}, d_{n+1}\right)$ and $\left(\mathscr{A}^{(n+1)}, d_{n+1}^{\prime}\right)$.

We now construct the DGA $\left(\mathscr{N}^{(n+1)}, \delta_{n+1}\right)$. Again, we define $\left(\mathscr{N}^{(n+1)}, \delta_{n+1}\right)$ to be of the form

$$
\mathscr{N}^{(n+1)}=\mathscr{N}^{(n)} \otimes_{\delta_{n+1}} \Lambda\left(Z^{n+1}\right)
$$

This time, however, we define the differential $\delta_{n+1}$ to satisfy the requirement of the lemma. Given $w \in Z^{n+1}$, if $d(w) \neq d(\beta)$ for any decomposable $\beta \in \mathscr{M}$ then we set $\delta_{n+1}(w)=\varphi_{n}(d(w))$. Otherwise, we put $\delta_{n+1}(w)=0$. Observe that $\left(\mathscr{N}^{(n+1)}, \delta_{n+1}\right)$ is a minimal DGA with the required property. To show that $\left(\mathscr{M}^{(n+1)}, d_{n+1}\right)$ and $\left(\mathscr{N}^{(n+1)}, \delta_{n+1}\right)$ are isomorphic we use the relationship between minimal DGAs and rational Postnikov towers described in $\S 2$.

Consider the principal fibrations $p_{n+1}: X_{n+1} \rightarrow X_{n}$ and $p_{n+1}^{\prime}: X_{n+1}^{\prime} \rightarrow$ $X_{n}$ with fibre $K\left(\left(Z^{n+1}\right)^{*}, n+1\right)$ corresponding to the inclusions $\mathscr{N}^{(n)} \hookrightarrow$ $\mathscr{N}^{(n+1)}$ and $\mathscr{N}^{(n)} \hookrightarrow \mathscr{A}^{(n+1)}$ respectively, as described in $\S 2$. The $k$-invariants for $p_{n+1}$ and $p_{n+1}^{\prime}$ correspond to the linear maps $\delta_{n+1}\left|Z^{n+1}, d_{n+1}^{\prime}\right| Z^{n+1} \in$ $\operatorname{Hom}\left(Z^{n+1}, H^{n+2}\left(\mathscr{N}^{(n)}, \delta_{n}\right)\right)$, respectively. But by construction, $\delta_{n+1}(w)$ and $d_{n+1}^{\prime}(w)$ are cohomologous in $\left(\mathscr{N}^{(n)}, \delta_{n}\right)$ for all $w \in Z^{n+1}$. It follows that there is a fibre homotopy equivalence (actually a bundle equivalence) $f: X_{n+1} \rightarrow$ $X_{n+1}^{\prime}$ between $p_{n+1}$ and $p_{n+1}^{\prime}$ which covers the identity of $X_{n}$. Applying the minimal model functor $\mathscr{M}$, we obtain a DGA isomorphism $\psi=\mathscr{M}(f)$ : $\mathscr{A}^{(n+1)} \rightarrow \mathscr{N}^{(n+1)}$ with $\psi \mid \mathscr{N}^{(n)}$ equal to the identity. We then set $\varphi_{n+1}=$ $\psi \circ \varphi_{n+1}^{\prime}$, and the induction step is complete. Finally we note that, from the construction, it is clear that if $(\mathscr{M}, d)$ is two-stage minimal then so is $(\mathscr{N}, \delta)$. 
We are now prepared to prove

Lemma 3.4. Let $X$ be a simply connected complex whose minimal model is a two-stage minimal DGA. Let $X_{n}$ be the nth Postnikov section of $X$. Suppose $H^{q}\left(X_{n}, \mathbb{Q}\right)$ is generated by decomposables for all $q$ and $n$ with $q>n$. Then $X$ is formal.

Proof. Let $(\mathscr{M}, d)$ be the minimal model of $X$. We use Lemma 2.3 to show that $(\mathscr{M}, d)$ is formal. By hypothesis, $(\mathscr{M}, d)$ is of the form $\mathscr{M}=\Lambda\left(Z_{0}\right) \otimes_{d} \Lambda\left(Z_{1}\right)$, with $d \mid Z_{0}=0$. By Lemma 3.3, we may assume that $d \mid Z_{1}$ is injective and that for all homogeneous $z \in Z_{1}, d(z) \neq d(\beta)$ for any decomposable $\beta \in \mathscr{M}$.

For each $i=2, \ldots, \infty$, let $N_{i}=Z_{1}^{i}$, the vector space of degree $i$ elements of $Z_{1}$. Since $d \mid Z_{0}=0$ and $d \mid Z_{1}$ is injective, $C_{i}=Z_{0}^{i}$ is the space of degree $i$ closed generators. Thus $N_{i}$ is a complement to $C_{i}$ for each $i$. To prove the lemma we must show that all closed elements in $I\left(Z_{1}\right)$ are exact.

Let $\operatorname{CL}\left(I\left(Z_{1}\right)\right)$ denote the set of all closed elements of $I\left(Z_{1}\right)$. We claim that each homogeneous element $\alpha \in \mathrm{CL}\left(I\left(Z_{1}\right)\right)$ is decomposable in $\mathscr{M}$. Otherwise, we may write $\alpha=z+\alpha^{\prime}$ where $z \in Z_{1}$ is homogeneous and $\alpha^{\prime} \in I\left(Z_{1}\right)$ is decomposable. But then, since $d(\alpha)=0$, we see that $d(z)=-d\left(\alpha^{\prime}\right)$, which contradicts our assumption above. Our claim implies that if $\alpha \in \operatorname{CL}\left(I\left(Z_{1}\right)\right)$ is homogeneous, say of degree $q$, then $\alpha \in \mathscr{M}^{(n)}$ for some $n<q$.

Now $\mathrm{CL}\left(I\left(Z_{1}\right)\right)$ is a multiplicatively closed subset of $\mathscr{M}$. Let $\mathrm{CL}\left(I\left(Z_{1}\right)\right)$. $\mathrm{CL}\left(I\left(Z_{1}\right)\right)$ denote the subspace of decomposables. Consider the quotient vector space

$$
\mathrm{CL}\left(I\left(Z_{1}\right)\right) /\left(\mathrm{CL}\left(I\left(Z_{1}\right)\right) \cdot \mathrm{CL}\left(I\left(Z_{1}\right)\right)\right) \text {. }
$$

Let $\rho$ be the projection of $\mathrm{CL}\left(I\left(Z_{1}\right)\right)$ onto this set, and choose a graded vector space $Q \subset \mathrm{CL}\left(I\left(Z_{1}\right)\right)$ such that $\rho \mid Q$ is a linear isomorphism. It is clear that we may choose $Q$ such that if $\alpha \in Q$ then no summand of $\alpha$ is in $\mathrm{CL}\left(I\left(Z_{1}\right)\right) \cdot \mathrm{CL}\left(I\left(Z_{1}\right)\right)$. Notice that sums and products of elements of $Q$ generate all elements of $\mathrm{CL}\left(I\left(Z_{1}\right)\right)$. Since sums and products of exact elements are exact, it suffices to show that all elements of $Q$ are exact.

Let $\alpha \in Q$. We may suppose $\alpha$ is homogeneous, say of degree $q$. As shown above, $\alpha \in \mathscr{M}^{(n)}$ for some $n<q$. Thus $\alpha$ is a closed element of the DGA $\left(\mathscr{M}^{(n)}, d_{n}\right)$. By hypothesis, $H^{q}\left(\mathscr{M}^{(n)}, d_{n}\right) \cong H^{q}\left(X_{n}, \mathbb{Q}\right)$ is additively generated by decomposable elements. Now the decomposable elements of $H^{q}\left(\mathscr{M}^{(n)}, d_{n}\right)$ are all represented by elements in the space $\Lambda\left(Z_{0}\right) \oplus \operatorname{CL}\left(I\left(Z_{1}\right)\right) \cdot \operatorname{CL}\left(I\left(Z_{1}\right)\right)$. Since $\alpha$ is not an element of this space, it must be cohomologous to such an element. Specifically, we see that there is some $\beta \in \mathscr{M}$ such that $d(\beta)=$ $\alpha+\alpha^{\prime}+\gamma$, where $\alpha^{\prime} \in \mathrm{CL}\left(I\left(Z_{1}\right)\right) \cdot \operatorname{CL}\left(I\left(Z_{1}\right)\right)$ and $\gamma \in \Lambda\left(Z_{0}\right)$. By Lemma 3.2, we may write $\beta=\beta_{0}+\beta_{1}$ such that $d\left(\beta_{0}\right) \in \Lambda\left(Z_{0}\right)$ and $d\left(\beta_{1}\right) \in I\left(Z_{1}\right)$. In particular, $d\left(\beta_{1}\right)=\alpha+\alpha^{\prime}$. Since $\alpha$ has no summands in $\operatorname{CL}\left(I\left(Z_{1}\right)\right) \cdot \operatorname{CL}\left(I\left(Z_{1}\right)\right)$, we see that actually $\alpha^{\prime}=0$ and $d\left(\beta_{1}\right)=\alpha$.

\section{THE MAIN RESUltS}

Our first main result removes the restriction placed on $X$ in Lemma 3.4. We prove

Theorem 4.1. Let $X$ be a simply connected complex and $X_{n}$ the nth Postnikov section of $X$. Suppose $H^{q}\left(X_{n}, \mathbb{Q}\right)$ is generated by decomposable elements for all $q$ and $n$ with $q>n$. Then $X$ is formal. 
Proof. Let $(\mathscr{M}, d)$ denote the minimal model of $X$. In view of Lemma 3.4, it suffices to show that $(\mathscr{M}, d)$ is isomorphic to a two-stage minimal DGA. As in the proof of Lemma 3.3, we construct a two-stage minimal DGA $(\mathscr{N}, \delta)$ and an isomorphism $\varphi: \mathscr{M} \rightarrow \mathscr{N}$, inductively. As before, we let $\mathscr{N}^{(2)}=\mathscr{M}^{(2)}$ and let $\varphi_{2}$ be the identity. As an induction hypothesis, we assume that a two-stage minimal DGA $\left(\mathscr{N}^{(n)}, \delta_{n}\right)$ and an isomorphism $\varphi_{n}: \mathscr{M}^{(n)} \rightarrow \mathscr{N}^{(n)}$ have been constructed such that $\mathscr{N}^{(n)}$ is generated by elements of degree $\leq n$. To prove the theorem, we must construct a two-stage minimal DGA $\left(\mathscr{N}^{(n+1)}, \delta_{n+1}\right)$ and a DGA isomorphism $\varphi_{n+1}: \mathscr{M}^{(n+1)} \rightarrow \mathscr{N}^{(n+1)}$ such that $\left(\mathscr{N}^{(n)}, \delta_{n}\right)$ is the subDGA of $\left(\mathscr{N}^{(n+1)}, \delta_{n+1}\right)$ generated by elements of degree $\leq n$ and such that $\varphi_{n+1} \mid \mathscr{M}^{(n)}=\varphi_{n}$.

As an algebra, $\mathscr{M}=\Lambda(Z)$ for some graded vector space $Z$. As DGA, by our induction hypothesis $\left(\mathscr{N}^{(n)}, \delta_{n}\right)$ is of the form $\mathscr{N}^{(n)}=\Lambda\left(W_{0}\right) \otimes_{\delta_{n}} \Lambda\left(W_{1}\right)$, where $W_{0}$ and $W_{1}$ are graded vector spaces with no elements in degree $>n$ and where $\delta_{n} \mid W_{0}=0$. By Lemma 3.3, we may suppose that $\delta_{n} \mid W_{1}$ is injective and that, given $w \in W_{1}, \quad \delta_{n}(w) \neq \delta_{n}(\beta)$ for any decomposable $\beta \in \mathscr{N}^{(n)}$.

The construction proceeds as in the proof of Lemma 3.3. We first construct a DGA $\left(\mathscr{A}^{(n+1)}, d_{n+1}^{\prime}\right)$ by letting $\mathscr{A}^{(n+1)}=\mathscr{N}^{(n)} \otimes_{d_{n+1}^{\prime}} \Lambda\left(Z^{n+1}\right)$, where $d_{n+1}^{\prime}(z)=\varphi_{n}(d(z))$, for all $z \in Z^{n+1}$. Notice that if we define $\varphi_{n+1}^{\prime}: \mathscr{M}^{(n+1)} \rightarrow$ $\mathscr{A}^{(n+1)}$ by letting $\varphi_{n+1}^{\prime}=\varphi_{n}$ on $\mathscr{M}^{(n)}$ and the identity on $Z^{n+1}$ then $\varphi_{n+1}^{\prime}$ is a DGA isomorphism.

Now let $W_{0}^{n+1}=\operatorname{ker}\left(d \mid Z^{n+1}\right)$, and let $W_{1}^{n+1}$ be any complement to $W_{0}^{n+1}$ in $Z^{n+1}$. Let $w \in W_{1}^{n+1}$. We claim that $d_{n+1}^{\prime}(w)$ is cohomologous to an element of $\Lambda\left(W_{0}\right)$. To see this, use the decomposition (3.1) to write $d_{n+1}^{\prime}(w)=\alpha+\beta$ where $\alpha \in \Lambda\left(W_{0}\right)$ and $\beta \in I\left(W_{1}\right)$. Since $(\mathscr{M}, d)$ is minimal, $d$ takes decomposable values. By its definition, $d_{n+1}^{\prime}$ does also, so, in particular, $\beta$ is decomposable. Thus $\beta \in I\left(W_{1}\right) \cap \mathscr{N}^{(n)}$. Observe also that $\beta$ is closed. Since $\left(\mathscr{N}^{(n)}, \delta_{n}\right) \cong$ $\left(\mathscr{C}^{(n)}, d_{n}\right)$, by our hypothesis on $X,\left(\mathscr{N}^{(n)}, \delta_{n}\right)$ satisfies the conditions of Lemma 3.4. By the proof of Lemma 3.4 we obtain that $\beta$ is exact. Thus $d_{n+1}^{\prime}(w)$ is cohomologous to $\alpha \in \Lambda\left(W_{0}\right)$.

We now construct $\left(\mathscr{N}^{(n+1)}, \delta_{n+1}\right)$. Let

$$
\mathscr{N}^{(n+1)}=\mathscr{N}^{(n)} \otimes_{\delta_{n+1}} \Lambda\left(Z^{n+1}\right),
$$

where we define $\delta_{n+1} \mid Z^{n+1}$ as follows. Given $w \in Z^{n+1}$, if $d_{n+1}^{\prime}(w) \in \Lambda\left(W_{0}\right)$ then we put $\delta_{n+1}(w)=d_{n+1}^{\prime}(w)$. Otherwise, by the previous paragraph $d_{n+1}^{\prime}(w)$ is cohomologous to some element $\alpha \in \Lambda\left(W_{0}\right)$. In this case we set $\delta_{n+1}(w)=\alpha$. Observe that, as defined, $\left(\mathscr{N}^{(n+1)}, \delta_{n+1}\right)$ is a two-stage minimal DGA. Observe also, that the linear maps $\delta_{n+1}\left|Z^{n+1}, d_{n+1}^{\prime}\right| Z^{n+1} \in \operatorname{Hom}\left(Z^{n+1}, H^{n+2}\left(\mathscr{N}^{(n)}, \delta_{n}\right)\right)$ coincide, since by construction $\delta_{n+1}(w)$ and $d_{n+1}^{\prime}(w)$ are cohomologous for all $w \in Z^{n+1}$. Just as in the proof of Lemma 3.3 we obtain a DGA isomorhism $\psi: \mathscr{A}^{(n+1)} \rightarrow \mathscr{N}^{(n+1)}$ such that $\psi \mid \mathscr{N}^{(n)}$ is the identity. Finally, set $\varphi_{n+1}=$ $\psi \circ \varphi_{n+1}^{\prime}$, and the proof is complete.

The following example shows that the implication in Theorem 4.1 cannot be reversed.

Example 4.2 (see [3, p. 261]). Let $X=S^{2} \vee \mathbb{C} P^{\infty}$. Then $X$ is a simply connected formal space, for $S^{2}$ and $\mathbb{C} P^{\infty}$ are formal spaces and wedges of formal 
spaces are formal by [5, Lemma 1.6]. Let $(\mathscr{M}, d)$ be the minimal model of $X$. Then it is clear that $\left(\mathscr{K}^{(3)}, d_{3}\right)$, the minimal model of the third Postnikov section $X_{3}$ of $X$, is given by

$$
\begin{gathered}
\mathscr{M}^{(3)}=\Lambda_{2}(x, y) \otimes_{d_{3}} \Lambda_{3}(\psi, \varphi), \\
d_{3}(x)=d_{3}(y)=0, \quad d_{3}(\psi)=x^{2}, \quad \text { and } \quad d_{3}(\varphi)=x \cdot y .
\end{gathered}
$$

Observe that the element $x \cdot \varphi+y \cdot \psi$ is a closed element of degree 5 in $\mathscr{M}^{(3)}$ which is not exact. Since there are no nontrivial decomposables in $H^{5}\left(X_{3}, \mathbb{Q}\right)=$ $H^{5}\left(\mathscr{M}^{(3)}, d_{3}\right), \quad x \cdot \varphi+y \cdot \psi$ represents an indecomposable in $H^{5}\left(X_{3}, \mathbb{Q}\right)$.

The example establishes that our condition involving decomposables in the rational cohomology of Postnikov sections is not, in general, equivalent to formality. The purpose of the remainder of this paper is to show that our condition is, in fact, equivalent to hyperformality.

The definition of hyperformality (Definition 2.2) for a space $X$ can be recast in terms of the minimal model of $X$. Specifically, we have

Lemma 4.3. Let $X$ be a simply connected complex. Then $X$ is hyperformal if and only if its minimal model $\left(\mathscr{M}(X), d_{X}\right)$ is of the form

$$
\mathscr{M}(X)=\Lambda\left(Z_{0}\right) \otimes_{d_{X}} \Lambda\left(Z_{1}\right),
$$

where the differential $d_{X}$ is given by $d_{X} \mid Z_{0}=0$ while $d_{X} \mid Z_{1}$ is some degree one linear map from $Z_{1}$ to the decomposable elements of $\Lambda\left(Z_{0}\right)$ such that there exists an (ordered) homogeneous basis $z_{1}, z_{2}, \ldots$ for $Z_{1}$, for which the elements $d_{X}\left(z_{1}\right), d_{X}\left(z_{2}\right), \ldots$ form a regular sequence in $\Lambda\left(Z_{0}\right)$. In this case, the graded space $Z_{0}$ corresponds to a space of indecomposable generators of $H^{*}(X, \mathbb{Q})$.

Proof. By [2, pp. 92-93], if $X$ is hyperformal then its minimal model is of the prescribed form. (See also $[4$, p. 580].)

Conversely, if $\left(\mathscr{M}(X), d_{X}\right)$ is as described in the statement then its cohomology is given by

$$
H^{*}\left(\mathscr{M}(X), d_{X}\right)=\Lambda\left(Z_{0}\right) / I\left(d_{X}\left(z_{1}\right), d_{X}\left(z_{2}\right), \ldots\right) .
$$

Since $H^{*}(X, \mathbb{Q})=H^{*}\left(\mathscr{M}(X), d_{X}\right), \quad X$ is hyperformal, by definition.

Using Lemma 4.3 we prove

Lemma 4.4. Let $X$ be a simply connected hyperformal complex with finitedimensional rational homotopy, i.e., $\sum_{p} \operatorname{dim}_{\mathbb{Q}}\left(\pi_{p}(X) \otimes \mathbb{Q}\right)<\infty$. Let $n$ be the dimension of the highest nontrivial rational homotopy group of $X$. Then $H^{q}(X, \mathbb{Q})$ is additively generated by decomposables for all $q>n$.

Proof. By the description of $\left(\mathscr{M}(X), d_{X}\right)$ in Lemma 4.3, the indecomposable generators of $H^{*}\left(\mathscr{M}(X), d_{X}\right)$ are represented by the elements of $Z_{0}$. Since the differential $d_{X}$ takes values in the decomposables of $\mathscr{M}(X)$, there are no nontrivial cohomology relations between elements of $Z_{0}$ and other elements of $\mathscr{M}(X)$. Thus every closed element of $\mathscr{M}(X)$ which represents an indecomposable cohomology class must have a nontrivial element of $Z_{0}$ as a summand. But now, by the Sullivan isomorphism [2, p. 65], since $\pi_{q}(X) \otimes \mathbb{Q}=0$ for $q>n, \mathscr{M}(X)$ is generated by elements of degree $\leq n$. Thus $Z_{0}^{q}=0$ for $q>n$. 
Theorem 4.5. Let $X$ be a simply connected complex with finite-dimensional rational homotopy groups in each degree. Then $X$ is hyperformal if and only if $X_{n}$ is hyperformal for all $n \geq 2$, where $X_{n}$ is the nth Postnikov section of $X$. Proof. For the proof we use the fact that given a finite regular sequence $\alpha_{1}, \ldots$, $\alpha_{q}$ of positive degree elements in a graded algebra $H$, any permutation of $\alpha_{1}, \ldots, \alpha_{q}$ is also a regular sequence. This fact can be deduced from [4, Lemma 2.4].

Suppose first that $X$ is hyperformal. Let $(\mathscr{M}, d)$ be the minimal model of $X$. Then, by Lemma 4.3, $(\mathscr{M}, d)$ is of the form $\mathscr{M}=\Lambda\left(Z_{0}\right) \otimes_{d} \Lambda\left(Z_{1}\right)$ where $d \mid Z_{0}=0$ and where there is some homogeneous basis $z_{1}, z_{2}, \ldots$ of $Z_{1}$ such that the sequence $d\left(z_{1}\right), d\left(z_{2}\right), \ldots$ is regular in $\Lambda\left(Z_{0}\right)$. Given a graded vector space $Z$ let us write $Z^{(n)}$ for the graded vector space consisting of all elements of $Z$ with degree $\leq n$. Then the minimal model $\left(\mathscr{M}^{(n)}, d_{n}\right)$ of $X_{n}$ is given by $\mathscr{M}^{(n)}=\Lambda\left(Z_{0}^{(n)}\right) \otimes_{d_{n}} \Lambda\left(Z_{1}^{(n)}\right)$. To show $X_{n}$ is hyperformal, it suffices to show that if $z_{i_{1}}, \ldots, z_{i_{k}}$ form a homogeneous basis for $Z_{1}^{(n)}$ then $d\left(z_{i_{1}}\right), \ldots, d\left(z_{i_{k}}\right)$ form a regular sequence in $\Lambda\left(Z_{0}^{(n)}\right)$. To prove this choose $q>>0$ so that the $z_{i_{j}}$ all appear in the list $z_{1}, \ldots, z_{q}$. Now $d\left(z_{1}\right), \ldots, d\left(z_{q}\right)$ is a regular sequence in $\Lambda\left(Z_{0}\right)$. Since permutations of finite regular sequences are regular, we may permute this sequence to a obtain a regular sequence with the same $q$ elements but whose first $k$ elements are $d\left(z_{i_{1}}\right), \ldots, d\left(z_{i_{k}}\right)$. It follows from the definition that $d\left(z_{i_{1}}\right), \ldots, d\left(z_{i_{k}}\right)$ is a regular sequence in $\Lambda\left(Z_{0}\right)$. From this we easily obtain that $d\left(z_{i_{1}}\right), \ldots, d\left(z_{i_{k}}\right)$ is also a regular sequence in $\Lambda\left(Z_{0}^{(n)}\right)$. Thus $X_{n}$ is hyperformal.

Conversely, suppose $X_{n}$ is hyperformal for each $n$. Then, regarding the minimal model $(\mathscr{K}, d)$ of $X$, we have that $\left(\mathscr{M}^{(n)}, d_{n}\right)$ is given as in the previous paragraph where there is some homogeneous basis $z_{1}, \ldots, z_{k}$ of $Z_{1}^{(n)}$ such that $d_{n}\left(z_{1}\right), \ldots d_{n}\left(z_{k}\right)$ is a regular sequence in $\Lambda\left(Z_{0}^{(n)}\right)$. (Note that the sequence is finite, since $X$ has finite-dimensional rational homotopy in each degree.) Using again that permutations of finite regular sequence of positive degree elements are regular, we may suppose that the $z_{i}$ appear in ascending order of degrees. With this assumption it is clear that the full sequence $d\left(z_{1}\right), d\left(z_{2}\right), \ldots$ corresponding to the homogeneous basis $z_{1}, z_{2}, \ldots$ of $Z_{1}$ is regular in $\Lambda\left(Z_{0}\right)$. It follows that $X$ is hyperformal.

Finally, we invoke the result of Felix and Halperin to conclude that our condition involving decomposables in the cohomology of Postnikov sections is equivalent to hyperformality.

Theorem 4.6. Let $X$ be a simply connected complex with finite-dimensional rational homotopy groups in each degree. Then $X$ is hyperformal if and only if $H^{q}\left(X_{n}, \mathbb{Q}\right)$ is additively generated by decomposables for all $q$ and $n$ with $q>n$.

Proof. Suppose $X$ is hyperformal. Then, by Theorem 4.5, $X_{n}$ is hyperformal for all $n$. By Lemma $4.4, H^{q}\left(X_{n}, \mathbb{Q}\right)$ is additively generated by decomposables for all $q>n$.

Conversely, if $H^{q}\left(X_{n}, \mathbb{Q}\right)$ is generated by decomposables for all $q$ and $n$ with $q>n$, then applying Theorem 4.1 to $X_{n}$ we have that $X_{n}$ is formal for each $n$. By our hypothesis on $X$, each $X_{n}$ is a simply connected formal 
complex with finite-dimensional rational homotopy. By [4, Theorem I], each $X_{n}$ is hyperformal. Thus, by Theorem $4.5, X$ is hyperformal.

Theorem 4.6 provides a quick, practical method for determining whether or not a given space is hyperformal. For instance, from Example 4.2 and the theorem we see that $S^{2} \vee \mathbb{C} P^{\infty}$ is a formal but not a hyperformal space. As another illustration of the method we establish the following:

Example 4.7. Let $X=\bigvee_{i=1}^{n} S^{k_{i}}$ be a wedge of $n$ odd-dimensional spheres. Then $X$ is hyperformal if and only if $n=1$.

As sufficiency is immediate we assume $n>1$ and prove $X$ is not hyperformal. Suppose first that the $k_{i}$ 's are all equal so that $X=\bigvee_{i=1}^{n} S^{k}$ for $k$ odd. Then we see that the minimal model $\left(\mathscr{M}^{(2 k-1)}, d_{2 k-1}\right)$ for the $(2 k-1)$ st Postnikov section, $X_{2 k-1}$, of $X$ is given by

$$
\begin{gathered}
\mathscr{M}^{(2 k-1)}=\Lambda_{k}\left(x_{1}, \ldots, x_{n}\right) \otimes_{d_{2 k-1}} \Lambda_{2 k-1}\left(y_{i j}\right), \quad 1 \leq i<j \leq n, \\
d_{2 k-1}\left(x_{i}\right)=0 \text { and } d_{2 k-1}\left(y_{i j}\right)=x_{i} \cdot x_{j} .
\end{gathered}
$$

Observe that the elements $x_{i} \cdot y_{i j}$ and $x_{j} \cdot y_{i j}$ represent indecomposable cohomology classes in $H^{3 k-1}\left(X_{2 k-1}, \mathbb{Q}\right) \cong H^{3 k-1}\left(\mathscr{M}^{(2 k-1)}, d_{2 k-1}\right)$. By Theorem 4.6, $X$ is not hyperformal.

Next suppose that $k_{1}<k_{2}<k_{3} \leq \cdots \leq k_{n}$. Let $k=k_{1}+k_{2}-1$. Then we have that $\left(\mathscr{M}^{(k)}, d_{k}\right)$, the minimal model for the $k$ th Postnikov section of $X$, is given by

$$
\begin{aligned}
& \mathscr{M}^{(k)}=\left(\bigotimes_{k_{i} \leq k} \Lambda_{k_{i}}\left(x_{i}\right)\right) \otimes_{d_{k}} \Lambda_{k}(y), \\
& d_{k}\left(x_{i}\right)=0 \text { and } d_{k}(y)=x_{1} \cdot x_{2} .
\end{aligned}
$$

In this case the elements $x_{1} \cdot y$ and $x_{2} \cdot y$ represent indecomposable cohomology classes in $H^{k+k_{1}}\left(X_{k}, \mathbb{Q}\right)$ and $H^{k+k_{2}}\left(X_{k}, \mathbb{Q}\right)$, respectively. Again by Theorem 4.6 we conclude that $X$ is not hyperformal. The remaining cases are handled similarly.

(Note. Halperin and Stasheff have shown [5, Theorem 1.5] that a wedge of odd spheres $X$ is "intrinsically formal"; that is, $X$ is a formal space with the property that $H^{*}(Y, \mathbb{Q}) \cong H^{*}(X, \mathbb{Q})$ implies $Y$ is rationally equivalent to $X$. The above example thus reconfirms the assertion [4, p. 576] that the class of hyperformal spaces is properly contained in the class of intrinsically formal spaces.)

Remark 4.8. In conclusion we remark that Theorem 4.6 offers a geometric answer to the algebraic problem of deciding whether or not a sequence in a graded algebra is regular.

\section{REFERENCES}

1. M. Arkowitz, Formal differential graded algebras and homomorphisms, J. Pure Appl. Algebra 51 (1988), 35-52.

2. A. K. Bousfield and V. K. A. M. Gugenheim, On PL de Rham theory and rational homotopy type, Mem. Amer. Math. Soc., no. 179, Amer. Math. Soc., Providence, RI, 1976. 
3. P. Deligne, P. Griffiths, J. Morgan, and D. Sullivan, Real homotopy theory of Kähler manifolds, Invent. Math. 29 (1975), 245-275.

4. Y. Felix and S. Halperin, Formal spaces with finite dimensional rational homotopy, Trans. Amer. Math. Soc, 270 (1982), 575-588.

5. S. Halperin and J. Stasheff, Obstructions to homotopy equivalences, Adv. Math. 32 (1979), 233-279.

6. I. Kaplansky, Commutative rings, Univ. Chicago Press, Chicago, 1974.

7. J. Neisendorfer and T. Miller, Formal and coformal spaces, Illinois J. Math. 22 (1978), 565-580.

8. J. Oprea, DGA homology decompositions and a condition for formality, Illinois J. Math. 30 (1986), 122-137.

9. D. Sullivan, Infinitesimal computations in topology, Inst. Hautes Études Sci. Publ. Math. 47 (1977), 269-331.

School of Mathematics, University of Minnesota, Minneapolis, Minnesota 55455

Current address: Department of Mathematics, Washington College, Chestertown, Maryland 21620

E-mail address: sam_smith.faculty@rashcoll.edu 